\title{
Polarization Characterization of Soft X-Ray Radiation at FERMI FEL-2
}

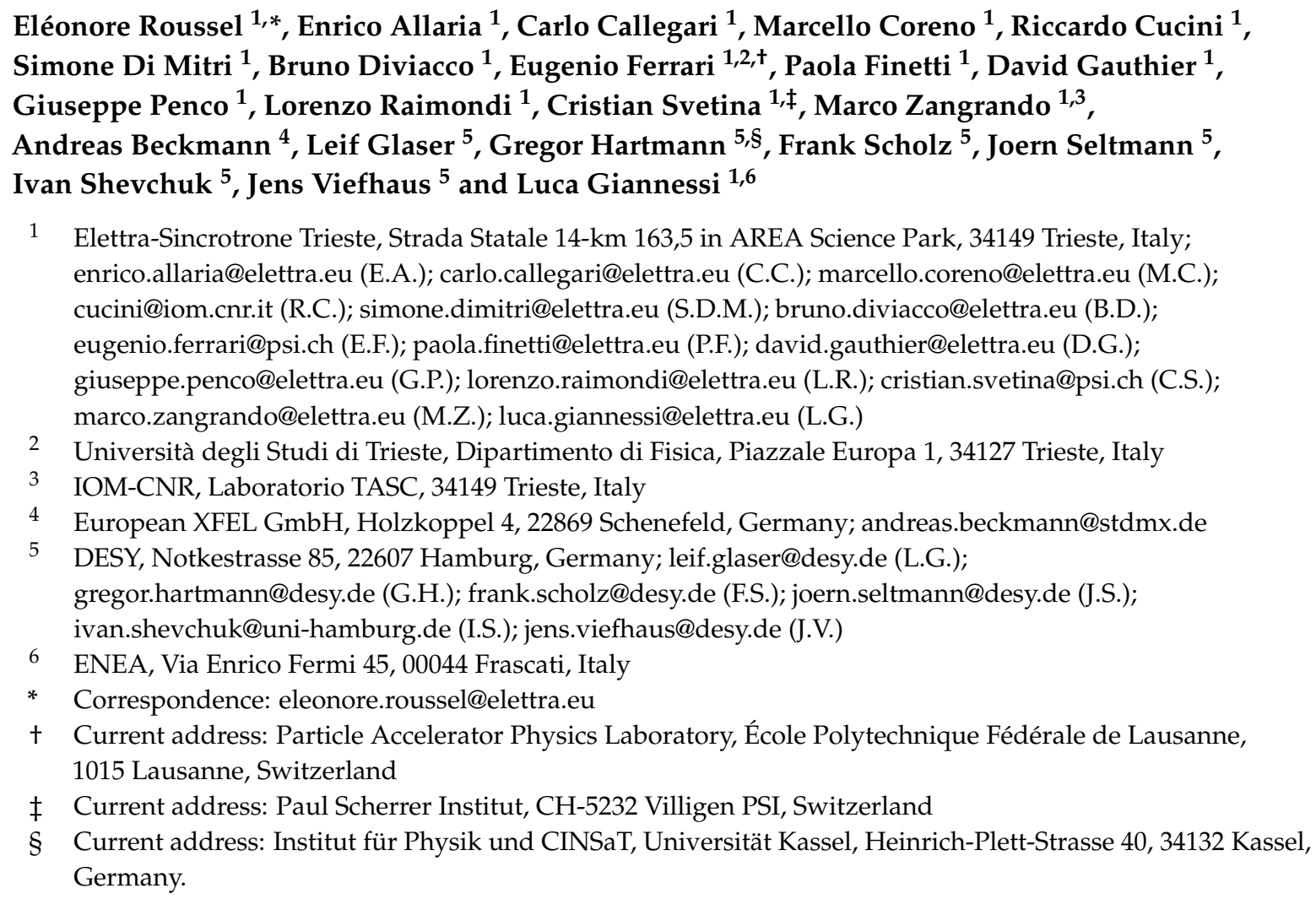

Received: 31 January 2017; Accepted: 5 April 2017; Published: 10 April 2017

\begin{abstract}
The control of polarization state in soft and hard X-ray light is of crucial interest to probe structural and symmetry properties of matter. Thanks to their Apple-II type undulators, the FERMI-Free Electron Lasers are able to provide elliptical, circular or linearly polarized light within the extreme ultraviolet and soft X-ray range. In this paper, we report the characterization of the polarization state of FERMI FEL-2 down to $5 \mathrm{~nm}$. The results show a high degree of polarization of the FEL pulses, typically above $95 \%$. The campaign of measurements was performed at the Low Density Matter beamline using an electron Time-Of-Flight based polarimeter.
\end{abstract}

Keywords: free-electron laser; extreme ultraviolet; soft X-ray; polarization control

\section{Introduction}

Circularly polarized light is a fundamental tool for studying structural and symmetry properties of matter with circular dichroism experiments. Circular dichroism enables investigations into the chirality of systems and the determination of magnetic properties [1-3] or specific molecular characteristics [4,5]. The use of highly intense, ultrashort elliptically polarized X-ray pulses offers the advantage of selective tunning to resonances, as well as the ability to probe on a small spatial scale, and on a femtosecond time scale, materials with tightly bound electrons. 
Variable-polarization undulators have long been used in synchrotron light sources to provide light of arbitrary polarization in the extreme ultraviolet and X-ray domain albeit with a long pulse duration (picoseconds) and a low peak power [6-11]. High-harmonic-generation (HHG) sources can easily produce ultrashort pulses and they can be adjusted to control the polarization ellipticity [12-14]. However, HHG pulses have a low intensity and are generally limited to the extreme ultraviolet range due to a sharp drop of efficiency of the conversion process.

The above limitations can be overcome by the new generation of soft and hard X-ray free electron lasers (XFEL). For several practical reasons, XFELs were designed, and currently operate, with linearly polarized undulators. Simplicity, lower cost of construction and tight requirements on the magnetic field quality favor linearly polarized undulators against variable polarization types. The former also offer higher magnetic fields in combination with a shorter undulator period. An alternative to produce a polarized hard X-ray beam by means of phase-retarder crystals has the drawbacks of a pulse intensity reduced by several orders of magnitude and of a poor degree of polarization, typically below $60 \%[15,16]$. Another alternative for radiation polarization control in facilities based on linearly polarized undulators relies on the superposition of two orthogonally polarized pulses [17-19]. Finally, in seeded FELs, where the amplification process is expedited by the use of an external seed, the required undulator length is reduced and the use of variable polarization undulators is a viable solution.

Among currently operational FELs, FERMI $[20,21]$ is the only one equipped with variable polarization undulators, of Apple-II type [22]. LCLS (Linac Coherent Light Source, USA) has recently commissioned a variable polarization afterburner of DELTA-type [23], placed at the end of the main undulator line [24]. However, such a design suffers from contamination by the linearly polarized FEL entering the afterburner and requires a delicate adjustment of the machine, e.g., reverse tapering and a beam-diverting scheme.

In the past years of FERMI operation, users have already exploited the circularly polarized light to study dichroic effects [3,25-27]. The purity of the polarization is a key parameter for the success of users' experiments. To satisfy this requirement, a systematic characterization of the polarization state of the FERMI FEL-1, whose wavelength ranges from 100 to $20 \mathrm{~nm}$, was conducted a few years ago [28] and has shown a very good degree of polarization, consistently above $90 \%$. More recently, a second FEL line (FERMI FEL-2) has been commissioned to produce radiation down to $4 \mathrm{~nm}$ and is now available for users' operation [21].

In this paper, we perform a systematic characterization of the polarization state of FERMI FEL-2: in Section 2.1, we briefly describe the FERMI FEL-2 line. Then, we present the experimental setup: the photon beam transport in Section 2.2 and the electron Time-Of-Flight (eTOF) based polarimeter in Section 2.3. Section 3 reports the results obtained and an evaluation of the effect of the transport optics. Section 4 summarizes our conclusions.

\section{Materials and Methods}

\subsection{FERMI FEL-2}

FERMI is a FEL user facility. It hosts two separate FEL lines (Figure 1) based on the external seeding technique, in order to deliver spatially and longitudinally coherent pulses in the extreme ultraviolet range with ultrahigh peak brightness, narrow bandwidth spectrum, and stable and ultra short pulse duration in the sub-100-fs range $[20,21,29]$. The FEL-1 line covers the spectral range $100-20 \mathrm{~nm}$, while the FEL-2 line can reach shorter wavelengths in the range $20-4 \mathrm{~nm}$, thanks to a double stage high gain harmonic generation (HGHG) setup [30] based on the fresh bunch approach [31]. The two lines share the electron beam delivered by the same linear accelerator complex. The electron beam energy can be adjusted from 0.9 to $1.5 \mathrm{GeV}$. In the case of FEL-2, this enables us to extend the tuning range depending on the required polarization state and wavelength. In this study, the characterisation of the second stage radiation at "long" wavelength, i.e., above $6 \mathrm{~nm}$, were performed 
at an electron beam energy of $1 \mathrm{GeV}$, while the measurements below $6 \mathrm{~nm}$ were done at an energy of $1.45 \mathrm{GeV}$. The electron beam parameters used in the experiment are reported in Table 1.

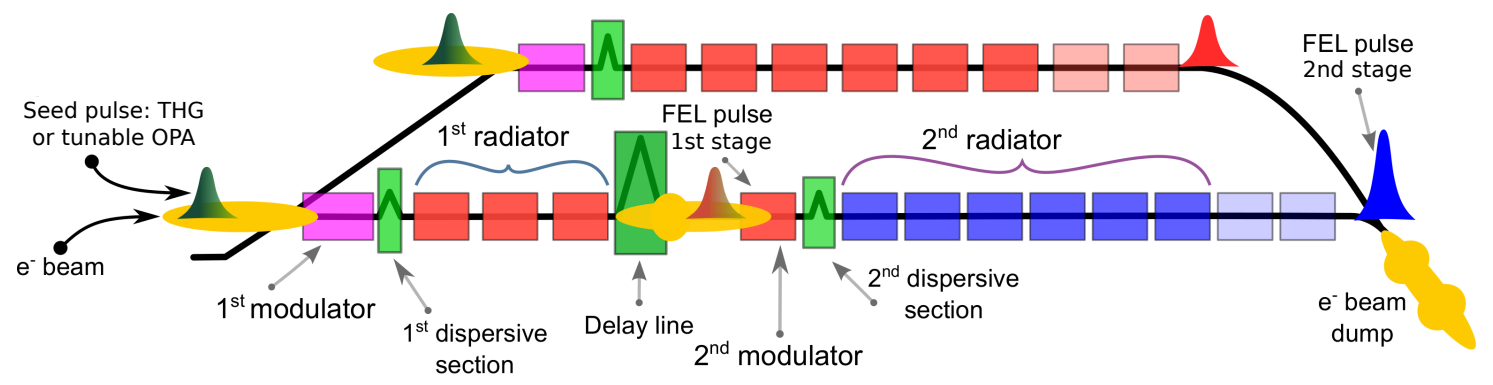

Figure 1. FERMI FELs layout: FEL-1 single-stage HGHG configuration (top) and FEL-2 double-stage cascaded HGHG configuration (bottom).

Table 1. Electron beam parameters used in the experiment.

\begin{tabular}{lcc}
\hline Parameter & Value & Unit \\
\hline Energy & $1.0 / 1.45$ & $\mathrm{GeV}$ \\
Slice energy spread (rms) & 150 & $\mathrm{keV}$ \\
Slice transverse normalized emittance & $\sim 1.5$ & $\mathrm{~mm} . \mathrm{mrad}$ \\
Peak current & 650 & $\mathrm{~A}$ \\
Average beam transverse size & 125 & $\mu \mathrm{m}$ \\
$\quad$ in the undulator (rms) & & \\
Electron beam magnetic delay line & 260 & $\mathrm{fs}$ \\
Repetition rate & 10 & $\mathrm{~Hz}$ \\
\hline
\end{tabular}

Compared with self-amplified spontaneous emission (SASE) mode, the HGHG scheme uses an external laser to imprint a modulation on the electron beam, which is then amplified and leads to coherent light emission at harmonics of the initial laser frequency. There are two configurations available for the seed laser of FERMI. A seed laser with tunable wavelength is provided by an optical parametric amplifier (OPA) pumped by a Ti:Sapphire laser, or a fixed-wavelength seed laser is obtained by the third harmonic generation (THG) of the Ti:Sapphire laser. The best output pulse energy performance and spectral quality are usually available when the FEL is seeded with the THG seed laser, which is the mode used for the campaign of polarization measurements on FEL-2. The THG seed laser parameters are summarized in Table 2.

Table 2. Third harmonic generation (THG) seed laser parameters used in the experiment.

\begin{tabular}{lcc}
\hline Parameter & Value & Unit \\
\hline Wavelength & 261.5 & $\mathrm{~nm}$ \\
Bandwidth (FWHM) & 1.3 & $\mathrm{~nm}$ \\
Pulse duration (FWHM) & 135 & $\mathrm{fs}$ \\
Available energy per pulse & $\leq 40$ & $\mu \mathrm{J}$ \\
Average beam size & 400 & $\mu \mathrm{m}$ \\
$\quad$ in the modulator (rms) & & \\
\hline
\end{tabular}

On FEL-2, the HGHG double-stage cascade scheme uses the light emitted by a first HGHG FEL to seed a second FEL stage (Figure 1, bottom). The interaction in the second stage is shifted to the head of the electron bunch by means of a magnetic chicane that delays the electron beam. Thus, the radiation of the first stage can seed a fresh portion of the electron beam that has not been affected by the FEL emission in the first stage. The first stage is a replica of the FEL-1 (Figure 1, top) with a reduced number of radiators. It is generally operated in circular polarization in order to obtain the 
highest energy per pulse necessary to seed the second stage but can also be set to linear horizontal or vertical polarization. The second stage can be tuned to linear horizontal, vertical or circular left or right polarization. As pointed out before, we used two different beam energies in order to extend the tuning range of the FEL. The associated parameters of the undulator chain are reported in Table 3.

In user operation, the light of the first stage is usually filtered out and the transport line of the FEL light is generally optimized for the short wavelengths emitted in the second part of the undulators. Moreover, the transverse profile of the first stage pulse is spoiled by diffraction in the vacuum chamber of the electron beam. Nonetheless, a recent user experiment took advantage of the presence of the first stage pulses in order to perform UV-pump soft-X-ray-probe either with the first or second stage pulse [32]. Another user experiment consisted of a soft-X-ray pump-probe experiment with both the first (pump) and the second (probe) stage [33]. The independent control of the polarization state of the first and second stage is thus of crucial interest for the user community.

Table 3. Main parameters of the FERMI FEL-2 undulators. The tuning ranges are given for an electron beam energy between 1.0 and $1.5 \mathrm{GeV}$. The polarization states are abbreviated as LH: linear horizontal, LV: linear vertical, CR: circular right, CL: circular left.

\begin{tabular}{lccc}
\hline & 1st Stage Modulator & $\begin{array}{c}\text { 1st Stage Radiators } \\
\text { and 2nd Stage Modulator }\end{array}$ & 2nd Stage Radiators \\
\hline Magnetic period (mm) & 100 & 55 & 35 \\
Tuning range (nm) & $300-200$ & $114-20$ & $18-4$ \\
Number of periods & 30 & 42 & 66 \\
Number of segments & 1 & $3+1$ & 6 \\
Polarization & $\mathrm{LH}$ & $\mathrm{LH}, \mathrm{LV}, \mathrm{CR}, \mathrm{CL}$ & $\mathrm{LH}, \mathrm{LV}, \mathrm{CR}, \mathrm{CL}$ \\
\hline
\end{tabular}

\subsection{The Photon Beam Transport}

The photon beam paths of the two FEL lines merge in the safety hutch (see Figure 2); photons are then transported to the experimental station through a common set of optics. The photon beam is analyzed before its delivery to the beamline endstations through the PADReS (Photon Analysis, Delivery and Reduction System) section of the machine [34]. Each FEL line has its own noninvasive beam diagnostics and transverse shaping instruments like an intensity monitor, a gas absorber and a set of double slits to adjust the beam aperture. At the end of the safety hutch, either the FEL-1 or the FEL-2 path can be selected by means of the PM1b plane mirror. At the exit of the safety hutch, the FEL spectrum is acquired using the energy spectrometer PRESTO (Pulse-Resolved Energy Spectrometer: Transparent and Online) [35] by diffracting and detecting $\approx 2 \%$ of the total intensity of the light. One of three different gratings can be used: the low-energy (LE) grating that covers the range 100-24.8 nm, the high-energy (HE) grating for the 27.6-6.7 nm range, and the super-high-energy (SHE) grating for wavelengths below $12.7 \mathrm{~nm}$. The almost unperturbed photon beam is transported to the desired endstation through the three-way chamber that deflects the beam by means of a switching mirror. The polarimeter used in the experiment was mounted on the Low Density Matter (LDM) endstation [36].

Due to the geometry of the optics transport to the LDM station (four mirrors reflecting in the horizontal plane and two in the vertical plane in the case of FEL-2), the vertical polarization is better transmitted than the horizontal one, especially for wavelengths longer than $10 \mathrm{~nm}$ (Figure 3a, red and blue lines). As an example, the ratio between the transmission of vertical and horizontal polarization for a radiation at $26 \mathrm{~nm}$ is equal to 1.1. Moreover, the transport optics contribute also to a different phase delay for the horizontal and the vertical polarization (Figure 3a, green line). The difference in transmission and phase delay induce a wavelength-dependent change of ellipticity. Figure $3 \mathrm{~b}$ shows the corresponding deformation of the photoelectron angular distribution for circularly polarized light at $26 \mathrm{~nm}$ (see Section 2.3 for details on the photoelectron angular distribution). 


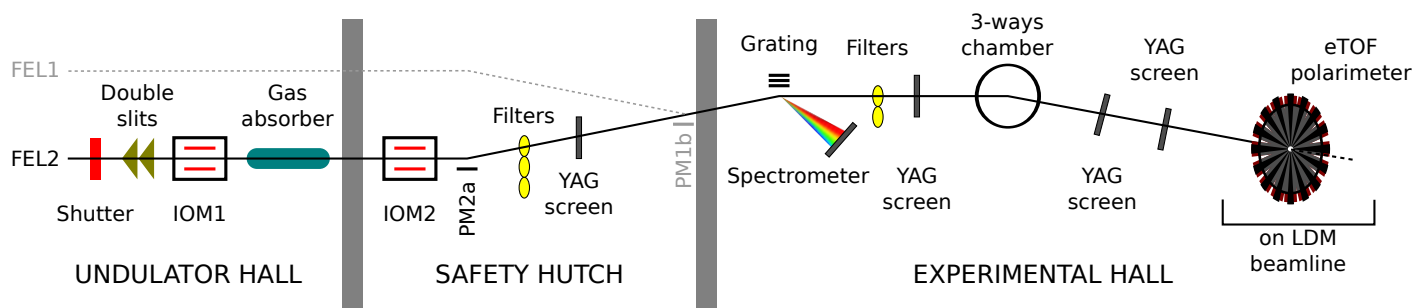

Figure 2. Schematic layout of the PADReS system to transport the light from FEL-2 to the LDM endstation where the eTOF polarimeter is installed. (IOM: ionization monitor, PM: plane mirror, YAG: Yttrium aluminium garnet).

(a)

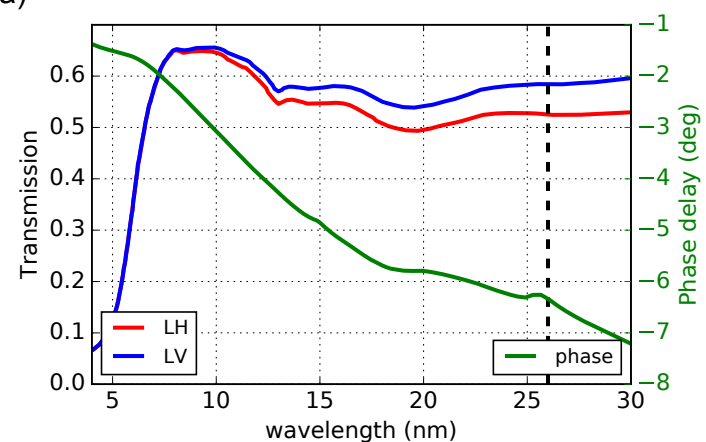

(b)

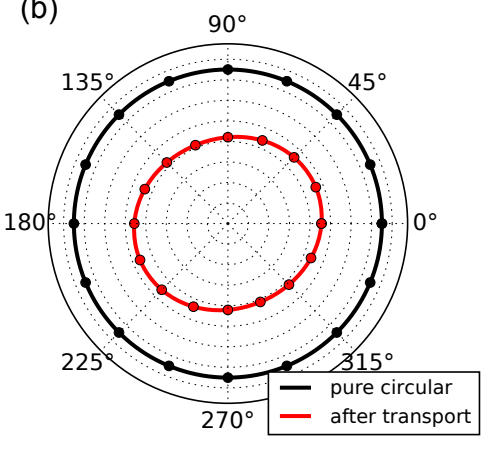

Figure 3. (a) calculated transmission of the LDM beamline optics for the FEL-2 line, for the horizontal linear polarization (red) and the vertical linear polarization (blue) [36] and simulated phase delay between vertical and horizontal polarization (green); (b) predicted photoelectron angular distribution in the case of a perfect circular polarization (black) and including the effect of the optical transport system (red) at $26 \mathrm{~nm}$ (dashed line in a).

In order to control the intensity ratio between the first and second stage, a series of filters (Zirconium, Palladium) can be inserted along the transport, in particular to suppress the radiation from the first stage. A gas cell can also be filled with diverse atomic gases to attenuate either the radiation of the first or second stage, depending on the selected gas (Nitrogen, Helium, Neon). A double slit system can be used as a spatial filter for the first stage light, which is more divergent than that of the second stage.

\subsection{The Electron Time-Of-Flight Polarimeter}

Several established methods exist to characterize the polarization of an undulator [27,37-40]. In our previous work [28], we extensively compared the merits of different instruments, namely, an optical, a fluorescence, and an eTOF polarimeter. We preferred the latter instrument over the other two because it permits non-invasive, single-shot measurements over a large wavelength range. In the case of FERMI FEL-2, the use of the eTOF polarimeter allows for simultaneous measurements of the two FEL stage emissions.

The eTOF polarimeter designed, manufactured and assembled by DESY in Hamburg, Germany, is a 16-channel electron time-of-flight (eTOF) spectrometer (Figure 2). The device is generally mounted such that it measures photoelectrons emitted in the plane perpendicular to the beam propagation direction $[41,42]$. The spectrometers allow us to detect the angular distribution of the photoelectrons that are emitted by the ionizing radiation of a target gas (here, He and $\mathrm{Ne}$ ). In our case, the kinetic energies of the detected electrons range from about $0-300 \mathrm{eV}$. The angular distribution depends on the degree of linear polarization and on the particular subshell of an atomic gas target that is ionized. 
In the dipole approximation, the photoelectron angular distribution (PAD) can be described by the following expression:

$$
P(\theta)=A\left\{1+\frac{\beta_{2}}{4}\left(1+3 P_{\text {lin }} \cos [2(\theta-\psi)]\right)\right\},
$$

where $A$ is the intensity, $P_{\text {lin }}$ the degree of linear polarization, $\psi$ the direction of the linear polarization and $\theta$ the angle of detection. Notice that $A \geq 0,0 \leq P_{\text {lin }} \leq 1$ and $-\pi \leq \psi \leq \pi$. $\beta_{2}$ describes the angular anisotropy parameter. For the case of He atoms, $\beta_{2}$ is equal to 2 , which leads, in the case of completely linear, horizontal polarized radiation, to an emission pattern with lobes in the horizontal direction and a node in the vertical direction.

The degree of circular polarization can be deduced upon assuming that the radiation is fully polarized. This assumption is reasonable in the case of FERMI FELs since it has already been demonstrated, by measuring the linear polarization, that the degree of polarization is clearly above $90 \%$ [28]. The degree of circular polarization $\left(P_{\text {circ }}\right)$, without distinction between left and right, can be evaluated by the formula $P_{\text {circ }}=\sqrt{1-P_{\text {lin }}^{2}}$.

The efficiency and the relative transmission of the 16 detectors were normalized by analyzing the distribution of a Ne gas target ionized at a wavelength of $43.7 \mathrm{~nm}$, where $\beta_{2}=0$ [43], i.e., the PAD is isotropic regardless of the state of light polarization. Only the first stage of FEL-2 was used for the calibration, in linear horizontal polarization at $43.7 \mathrm{~nm}$ with an energy per pulse of $\sim 2 \mu \mathrm{J}$ to avoid saturation effects in the detectors.
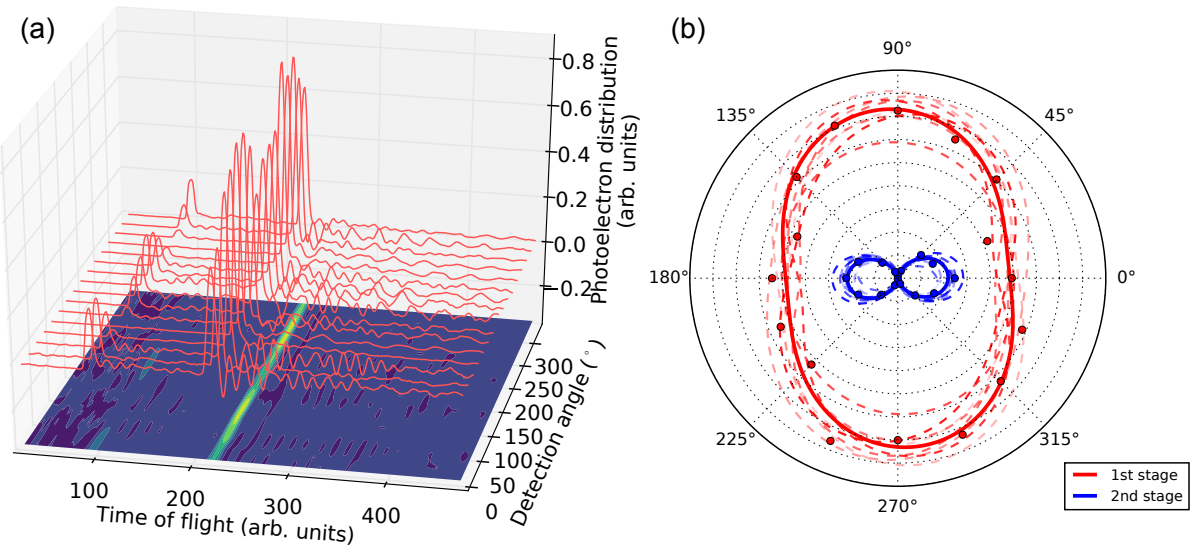

Figure 4. (a) photon energy spectra from the 16 channels of the eTOF polarimeter (average over 50 shots). The peak around 200 (arb. units) along the time-of-flight axis corresponds to the emission from the first stage and the peak around 100 (arb. units) corresponds to the second stage emission; (b) processed photon angular distribution of the first stage (red) and the second stage (blue) radiation. The dashed curves are single shot PADs while the solid line is an average over 50 shots. The lines are results from the fitting of data (points) with Equation (1).

Figure 4 shows a typical signal obtained from the eTOF polarimeter using He as gas target. The 16 spectral traces (Figure 4a) are composed of two photoelectron peaks. The calibration from time-of-flight $t$ to photon energy $E_{\mathrm{ph}}$ was done by fitting the FEL peaks to the formula $E_{\mathrm{ph}}=\left(a /\left(t-t_{0}\right)\right)^{2}+b$. This calibration allows for identifying the emission from the first and second stage but does not enter in the data processing. The photoelectron peak at long time-of-flight corresponds to the emission from the first stage, while the peak at short time-of-flight corresponds to the emission from the second stage. The analysis of the eTOF signals allows for reconstructing the angular distribution of the photoelectrons ionized by the two-color polarized FEL pulses (Figure 4b). The single-shot PAD is obtained by integrating numerically the area of the emission line of each detector. The intensity of the FEL pulses was adjusted in order to avoid saturation on the detectors and signal ringing, which can introduce cross-talk between the two stage signals. By fitting the single-shot PAD with Equation (1), 
one can determine the characteristics of the polarized light. In Figure 4, the two colors have different PAD patterns. Here, the first stage was set to circular right polarization while the second stage was set to linear horizontal one. For horiziontally polarized light, one can see the nodes along the vertical direction $\left(90^{\circ}, 270^{\circ}\right)$ in the photoelectron distribution.

\section{Results and Discussion}

Polarization measurements were carried out at the LDM beamline for various undulators configurations. Thanks to the parallel detection of electrons of all kinetic energies in the time-of-flight spectrometers and the single-shot capability of the eTOF polarimeter, it has been possible to measure simultaneously the polarization of the first and second stage of FEL-2 (Figure 5). A full set of acquisitions took around $10 \mathrm{~min}$, which corresponds to $\approx 6000$ FEL pulses. For the reported data, the first stage was tuned at the tenth and eleventh harmonic of the seed laser while the second stage was tuned at the fourth, fifth and sixth harmonic of the first stage. Figure 5 presents polarization data in a typical configuration of operation of FEL-2 where the first and second stage radiators are tuned to a different polarization state. The first stage (h10) produced circularly polarized light while the second stage $($ h10 $\times 5)$ emitted linearly horizontal polarized light. The eTOF polarimeter permits, for single shot analysis only, to measure the degree of linear polarization $\left(P_{\text {lin }}\right)$. Assuming a fully polarized light (with no unpolarized contributions), one can deduce the degree of circular polarization $\left(P_{\text {circ }}\right)$ of the first stage to be equal to 0.96 .
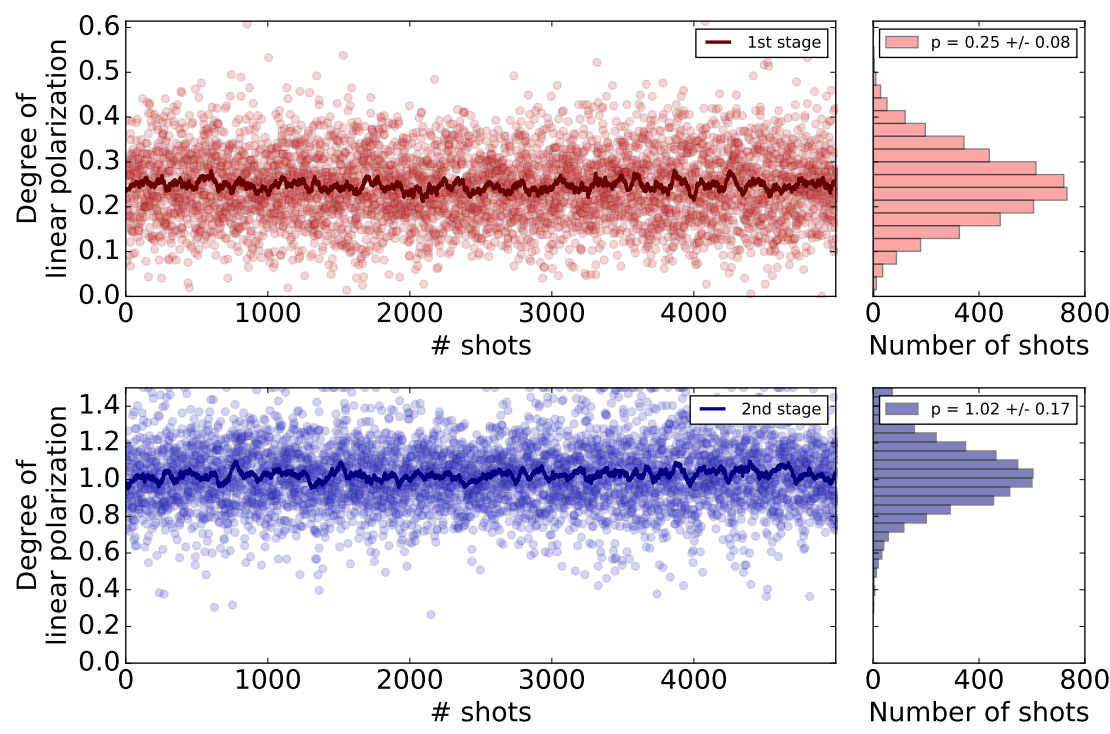

Figure 5. Degree of linear polarization $P_{\text {lin }}$ of the first stage (red) and of the second stage (blue). The undulators of the first stage were tuned in circular right polarization at $26.1 \mathrm{~nm}$ and the second stage was tuned in linear horizontal polarization at $5.22 \mathrm{~nm}$. The deduced degree of circular polarization is $P_{\text {circ }}=0.96$. The lines represent the moving average over 50 shots.

In the case of FEL-2, the light emitted from the first stage is used as a seed for the second stage. Inasmuch as the first and the second stage can be detected simultaneously in the eTOF polarimeter, we can study the influence of the first stage emission on the polarization state of the second stage (Figure 6). The intensity of both the first and second stage, inside the polarimeter (i.e., after transport to LDM through the PADReS line), is clearly correlated as expected (Figure 6a). From the data in Figure 6b, one can see that the degree of polarization of the second stage is independent of the polarization state of the first stage. Moreover, as shown in Figure $6 c, d$, the degree of polarization of the second stage is also insensitive to the intensity of the first stage seed as well as to the FEL output power (i.e., intensity of 
the second stage emission). As expected, the absence of correlation between the degree of polarization of the two stages and the intensities confirms that two polarizations are independent and can be tuned separately. Results also suggest, as already reported $[19,28]$, that measured polarization fluctuations are mostly related to instrumental statistical uncertainty of the polarimeter and not associated with a real fluctuation of the degree of polarization.
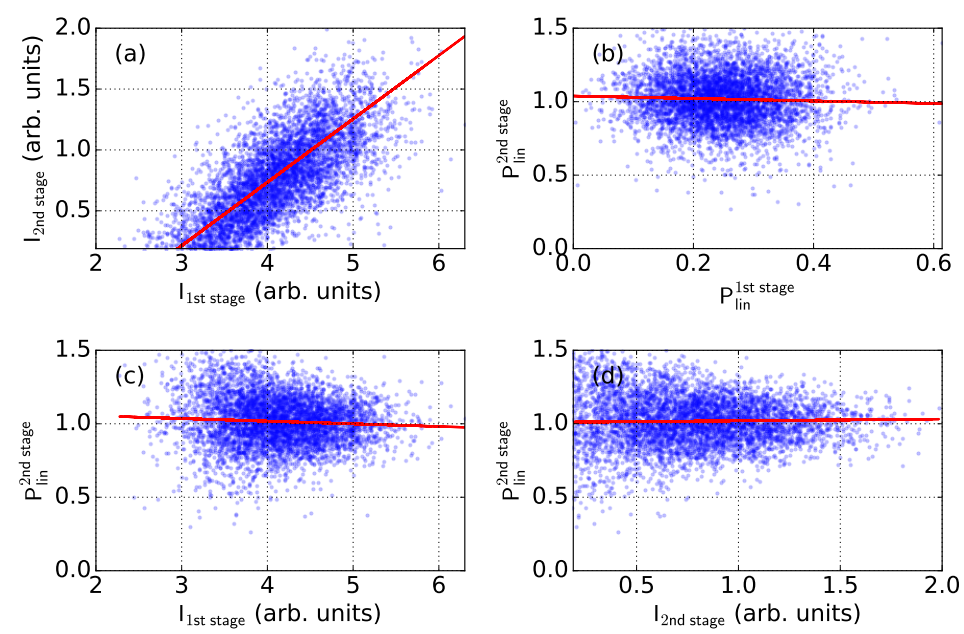

Figure 6. Correlation between first and second stage emission: intensity inside the eTOF polarimeter of the second stage in function of the first stage (a); degree of linear polarization of the second stage as a function of the first stage (b); and degree of linear polarization of the second stage in function of the intensity of the first (c) and the second (d) stage. Same data as in Figure 5. (blue: experimental data, red: linear regression).

One feature of FERMI FEL resides in the used undulator type, the variable polarization undulators of Apple-II type [22], which permits switching from one state to another state of polarization in a fast and reliable way. We report, in Figure 7, measurements of the direction of polarization with the radiators of the second stage tuned in linear horizontal (LH) and linear vertical (LV) polarization mode. The trend of the moving averages show a good stability of the direction of polarization and low single shot fluctuations.
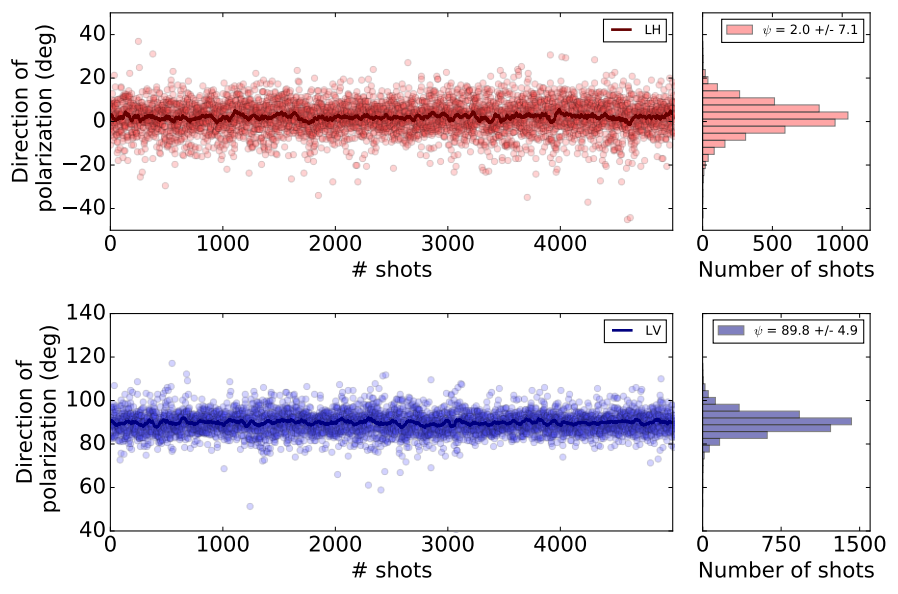

Figure 7. Direction of polarization $\psi$ of the second stage in the case of linear horizontal polarized light (red) and linear vertical polarized light (blue) at $5.22 \mathrm{~nm}$. The lines represent the moving average over 50 shots. 
The complete results of polarization measurements are summarized in Table 4. The Stokes parameters $S_{1}$ and $S_{2}$ are extracted from the degree and the direction of the linear polarization using trigonometric relationships, while $S_{3}$ is deduced assuming a completely polarized light. The results indicate a high degree of polarization $(>95 \%$ ) for almost all the polarizations and wavelengths studied. Figure 8 presents the measured Stokes parameters in the case of circularly polarized light, combined with the predicted Stokes parameters including beam-line-transport and contamination effects. In the case of circularly polarized light, we observe a non-negligible ellipticity that can be explained by the asymmetric transmission of the transport optics between LH and LV polarizations (Figure 3). The effect is particularly noticeable for "long" wavelengths. The calculation of the Stokes parameters neglects the unpolarized component of light that might be attributed to transport optics with residual roughness and diffraction effects. The carbon contamination can alter the transmission ratio between horizontal and vertical polarization [44] more than what was predicted from uncontaminated optics and reported in Figure 3a. The off-axis emission may also contribute to the degradation of the degree of polarization.

Table 4. Summary of the polarization measurements for various configurations of FEL-2 and deduced Stokes parameters $S_{1}, S_{2}$ and $S_{3}$.

\begin{tabular}{ccccccc}
\hline Wavelength $(\mathbf{n m})$ & FEL Polarization & $\boldsymbol{P}_{\text {lin }}$ & $\psi\left({ }^{\circ}\right)$ & $S_{\mathbf{1}} / S_{\mathbf{0}}$ & $S_{\mathbf{2}} / S_{\mathbf{0}}$ & $S_{\mathbf{3}} / S_{\mathbf{0}}$ \\
\hline 26.1 & Circular right & $0.27 \pm 0.08$ & $93.3 \pm 12.0$ & -0.24 & -0.03 & 0.96 \\
23.73 & Circular right & $0.25 \pm 0.13$ & $92.5 \pm 28.0$ & -0.17 & -0.03 & 0.96 \\
10.88 & Circular right & $0.31 \pm 0.09$ & $75.0 \pm 10.0$ & -0.25 & 0.15 & 0.95 \\
8.7 & Circular right & $0.34 \pm 0.09$ & $72.6 \pm 9.4$ & -0.26 & 0.18 & 0.94 \\
5.93 & Circular right & $0.20 \pm 0.09$ & $88.3 \pm 19.9$ & -0.17 & 0.01 & 0.97 \\
5.22 & Circular right & $0.26 \pm 0.12$ & $87.3 \pm 23.3$ & -0.20 & 0.02 & 0.96 \\
\hline 10.88 & Linear horizontal & $0.99 \pm 0.10$ & $6.8 \pm 3.7$ & 0.95 & 0.23 & 0.37 \\
8.7 & Linear horizontal & $1.00 \pm 0.12$ & $7.0 \pm 4.6$ & 0.95 & 0.24 & 0.40 \\
5.22 & Linear horizontal & $1.01 \pm 0.16$ & $2.2 \pm 6.2$ & 0.99 & 0.08 & 0.44 \\
\hline 8.7 & Linear vertical & $0.99 \pm 0.11$ & $84.7 \pm 4.0$ & -0.96 & 0.18 & 0.39 \\
5.22 & Linear vertical & $0.96 \pm 0.15$ & $89.9 \pm 5.1$ & -0.95 & 0.00 & 0.46 \\
\hline
\end{tabular}

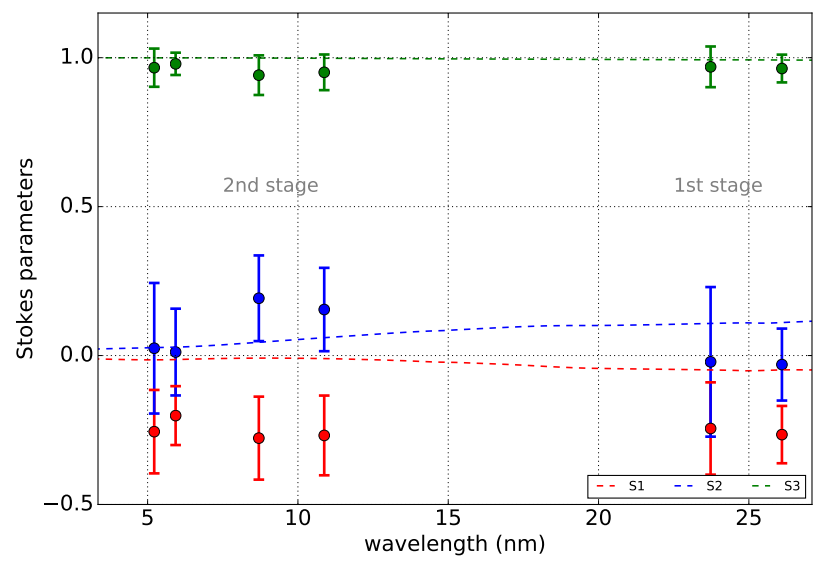

Figure 8. Impact of the LDM transport optics on the Stokes parameters (red $=S_{1} / S_{0}$, blue $=S_{2} / S_{0}$ and green $=S_{3} / S_{0}$ ) for a circularly polarized light. (dashed lines: predicted Stokes parameters; points: experimental data).

\section{Conclusions}

We have presented a study of the FEL polarization produced by FERMI FEL-2 in the soft X ray domain, for a large range of wavelengths and different undulator settings. The characterization 
of the second stage of FERMI FEL-2, together with the first stage, has been achieved. Our results highlight a high quality of the FEL polarization. We have shown that the polarization state of the two stages may be controlled and adjusted separately by changing the undulator polarization settings. These results pave the way for a new class of circular dichroism experiments based on two polarized beams. In the case of circular FEL polarization, a small ellipticity has been measured and may be explained, in part, by the asymmetry in the transmission of the $s$ and $p$ polarization along the photon transport. A fine tuning of the undulator settings could be used to compensate the effect of beamline transmission, thereby eliminating the spurious amount of linear polarization, in order to provide FEL radiation that is completely circularly polarized.

Acknowledgments: The authors are grateful to the entire FERMI team for the valuable support and operation of the machine.

Author Contributions: E.R., E.A., E.F. and G.P. coordinated the experiment. A.B., L.Gl., G.H., F.S., J.S., I.S. and J.V. designed, manufactured and assembled the eTOF polarimeter. C.C., M.C., R.C. and P.F. installed the polarimeter on LDM. L.R., C.S. and M.Z. transported and focused the soft X-ray beam in the polarimeter. C.S. performed the theoretical calculation of the Stokes parameters. E.R., E.A., S.D.M., B.D., E.F., D.G., G.P. and L.Gi. optimized the FEL during the preparation and execution of the experiment. E.R., E.F., E.A. and G.P. acquired the FEL polarization data and E.R. carried out data analysis, with contribution from G.H.. E.R. wrote the manuscript with contributions from all authors.

Conflicts of Interest: The authors declare no conflict of interest.

\section{References}

1. Eisebitt, S.; Luning, J.; Schlotter, W.F.; Lorgen, M.; Hellwig, O.; Eberhardt, W.; Stohr, J. Lensless imaging of magnetic nanostructures by X-ray spectro-holography. Nature 2004, 432, 885-888.

2. Boeglin, C.; Beaurepaire, E.; Halte, V.; Lopez-Flores, V.; Stamm, C.; Pontius, N.; Durr, H.A.; Bigot, J.Y. Distinguishing the ultrafast dynamics of spin and orbital moments in solids. Nature 2010, 465, 458-461.

3. Von Korff Schmising, C.; Pfau, B.; Schneider, M.; Günther, C.M.; Giovannella, M.; Perron, J.; Vodungbo, B.; Müller, L.; Capotondi, F.; Pedersoli, E.; et al. Imaging Ultrafast Demagnetization Dynamics after a Spatially Localized Optical Excitation. Phys. Rev. Lett. 2014, 112, 217203.

4. Garcia, G.A.; Nahon, L.; Lebech, M.; Houver, J.C.; Dowek, D.; Powis, I. Circular dichroism in the photoelectron angular distribution from randomly oriented enantiomers of camphor. J. Chem. Phys. 2003, $119,8781-8784$.

5. Janssen, M.H.M.; Powis, I. Detecting chirality in molecules by imaging photoelectron circular dichroism. Phys. Chem. Chem. Phys. 2014, 16, 856-871.

6. Desiderio, D.; Difonzo, S.; Diviacco, B.; Jark, W.; Krempasky, J.; Krempaska, R.; Lama, F.; Luce, M.; Mertins, H.C.; Placentini, M.; et al. The ELETTRA circular polarization beamline and electromagnetic elliptical wiggler insertion device. Synchrotron Radiat. News 1999, 12, 34-38.

7. Bahrdt, J.; Frentrup, W.; Gaupp, A.; Scheer, M.; Gudat, W.; Ingold, G.; Sasaki, S. Elliptically polarizing insertion devices at BESSY II. Nucl. Instrum. Methods Phys. Res. Sect. A 2001, 467-468, 21-29.

8. Benabderrahmane, C.; Berteaud, P.; Briquez, F.; Couprie, M.E.; Chubar, O.; Dubois, L.; Filhol, J.M.; Girault, M.; Level, M.P.; Marcouillé, O.; et al. First SOLEIL insertion devices are ready to produce photons for users. Nucl. Instrum. Methods Phys. Res. Sect. A 2007, 575, 33-37.

9. Nahon, L.; de Oliveira, N.; Garcia, G.A.; Gil, J.F.; Pilette, B.; Marcouillé, O.; Lagarde, B.; Polack, F. DESIRS: A state-of-the-art VUV beamline featuring high resolution and variable polarization for spectroscopy and dichroism at SOLEIL. J. Synchrotron Radiat. 2012, 19, 508-520.

10. Wang, H.; Bencok, P.; Steadman, P.; Longhi, E.; Zhu, J.; Wang, Z. Complete polarization analysis of an APPLE II undulator using a soft X-ray polarimeter. J. Synchrotron Radiat. 2012, 19, 944-948.

11. Viefhaus, J.; Scholz, F.; Deinert, S.; Glaser, L.; Ilchen, M.; Seltmann, J.; Walter, P.; Siewert, F. The Variable Polarization XUV Beamline P04 at PETRA III: Optics, mechanics and their performance. Nucl. Instrum. Methods Phys. Res. Sect. A 2013, 710, 151-154.

12. Vodungbo, B.; Sardinha, A.B.; Gautier, J.; Lambert, G.; Valentin, C.; Lozano, M.; Iaquaniello, G.; Delmotte, F.; Sebban, S.; Lüning, J.; et al. Polarization control of high order harmonics in the EUV photon energy range. Opt. Express 2011, 19, 4346-4356. 
13. Fleischer, A.; Kfir, O.; Diskin, T.; Sidorenko, P.; Cohen, O. Spin angular momentum and tunable polarization in high-harmonic generation. Nat. Photon. 2014, 8, 543-549.

14. Lambert, G.; Vodungbo, B.; Gautier, J.; Mahieu, B.; Malka, V.; Sebban, S.; Zeitoun, P.; Luning, J.; Perron, J.; Andreev, A.; et al. Towards enabling femtosecond helicity-dependent spectroscopy with high-harmonic sources. Nat. Commun. 2015, 6, 6167.

15. Pfau, B.; Günther, C.M.; Könnecke, R.; Guehrs, E.; Hellwig, O.; Schlotter, W.F.; Eisebitt, S. Magnetic imaging at linearly polarized X-ray sources. Opt. Express 2010, 18, 13608-13615.

16. Wang, T.; Zhu, D.; Wu, B.; Graves, C.; Schaffert, S.; Rander, T.; Müller, L.; Vodungbo, B.; Baumier, C.; Bernstein, D.P.; et al. Femtosecond Single-Shot Imaging of Nanoscale Ferromagnetic Order in Co/Pd Multilayers using resonant X-ray holography. Phys. Rev. Lett. 2012, 108, 267403.

17. Kim, K.J. Circular polarization with crossed-planar undulators in high-gain FELs. Nucl. Instrum. Methods Phys. Res. Sect. A 2000, 445, 329-332.

18. Deng, H.; Zhang, T.; Feng, L.; Feng, C.; Liu, B.; Wang, X.; Lan, T.; Wang, G.; Zhang, W.; Liu, X.; et al. Polarization switching demonstration using crossed-planar undulators in a seeded free-electron laser. Phys. Rev. Spec. Top. Accel. Beams 2014, 17, 020704.

19. Ferrari, E.; Allaria, E.; Buck, J.; De Ninno, G.; Diviacco, B.; Gauthier, D.; Giannessi, L.; Glaser, L.; Huang, Z.; Ilchen, M.; et al. Single Shot Polarization Characterization of XUV FEL Pulses from Crossed Polarized Undulators. Sci. Rep. 2015, 5, 13531.

20. Allaria, E.; Appio, R.; Badano, L.; Barletta, W.A.; Bassanese, S.; Biedron, S.G.; Borga, A.; Busetto, E.; Castronovo, D.; Cinquegrana, P.; et al. Highly coherent and stable pulses from the FERMI seeded free-electron laser in the extreme ultraviolet. Nat. Photon. 2012, 6, 699-704.

21. Allaria, E.; Castronovo, D.; Cinquegrana, P.; Craievich, P.; Dal Forno, M.; Danailov, M.B.; D'Auria, G.; Demidovich, A.; De Ninno, G.; Di Mitri, S.; et al. Two-stage seeded soft-X-ray free-electron laser. Nat. Photon. 2013, 7, 913-918.

22. Sasaki, S. Analyses for a planar variably-polarizing undulator. Nucl. Instrum. Methods Phys. Res. Sect. A 1994, 347, 83-86.

23. Temnykh, A.B. Delta undulator for Cornell energy recovery linac. Phys. Rev. Spec. Top. Accel. Beams 2008, $11,120702$.

24. Lutman, A.A.; MacArthur, J.P.; Ilchen, M.; Lindahl, A.O.; Buck, J.; Coffee, R.N.; Dakovski, G.L.; Dammann, L.; Ding, Y.; Dürr, H.A.; et al. Polarization control in an X-ray free-electron laser. Nat. Photon. 2016, 10, 468-472.

25. Capotondi, F.; Pedersoli, E.; Mahne, N.; Menk, R.H.; Passos, G.; Raimondi, L.; Svetina, C.; Sandrin, G.; Zangrando, M.; Kiskinova, M.; et al. Coherent imaging using seeded free-electron laser pulses with variable polarization: First results and research opportunities. Rev. Sci. Instrum. 2013, 84, 051301.

26. Müller, L.; Schleitzer, S.; Gutt, C.; Pfau, B.; Schaffert, S.; Geilhufe, J.; von Korff Schmising, C.; Schneider, M.; Günther, C.M.; Büttner, F.; et al. Ultrafast Dynamics of Magnetic Domain Structures Probed by Coherent Free-Electron Laser Light. Synchrotron Radiat. News 2013, 26, 27-32.

27. Mazza, T.; Ilchen, M.; Rafipoor, A.J.; Callegari, C.; Finetti, P.; Plekan, O.; Prince, K.C.; Richter, R.; Danailov, M.B.; Demidovich, A.; et al. Determining the polarization state of an extreme ultraviolet free-electron laser beam using atomic circular dichroism. Nat. Commun. 2014, 5, 3648.

28. Allaria, E.; Diviacco, B.; Callegari, C.; Finetti, P.; Mahieu, B.; Viefhaus, J.; Zangrando, M.; De Ninno, G.; Lambert, G.; Ferrari, E.; et al. Control of the Polarization of a Vacuum-Ultraviolet, High-Gain, Free-Electron Laser. Phys. Rev. X 2014, 4, 041040.

29. Allaria, E.; Badano, L.; Bassanese, S.; Capotondi, F.; Castronovo, D.; Cinquegrana, P.; Danailov, M.B.; D'Auria, G.; Demidovich, A.; De Monte, R.; et al. The FERMI free-electron lasers. J. Synchrotron Radiat. 2015, 22, 485-491.

30. Yu, L.H. Generation of intense uv radiation by subharmonically seeded single-pass free-electron lasers. Phys. Rev. A 1991, 44, 5178-5193.

31. Ben-Zvi, I.; Yang, K.; Yu, L. The "fresh-bunch" technique in FELS. Nucl. Instrum. Methods Phys. Res. Sect. A 1992, 318, 726-729.

32. Piseri, P. Dipartimento di Fisica, Università degli Studi di Milano, Milano, Italy. Private communication, 2016.

33. Fennel, T. Institute of Physics, University of Rostock, Rostock, Germany. Private communication, 2016. 
34. Zangrando, M.; Cocco, D.; Fava, C.; Gerusina, S.; Gobessi, R.; Mahne, N.; Mazzucco, E.; Raimondi, L.; Rumiz, L.; Svetina, C. Recent results of PADReS, the Photon Analysis Delivery and REduction System, from the FERMI FEL commissioning and user operations. J. Synchrotron Radiat. 2015, 22, 565-570.

35. Svetina, C.; Cocco, D.; Mahne, N.; Raimondi, L.; Ferrari, E.; Zangrando, M. PRESTO, the on-line photon energy spectrometer at FERMI: Design, features and commissioning results. J. Synchrotron Radiat. 2016, 23, 35-42.

36. Svetina, C.; Grazioli, C.; Mahne, N.; Raimondi, L.; Fava, C.; Zangrando, M.; Gerusina, S.; Alagia, M.; Avaldi, L.; Cautero, G.; et al. The Low Density Matter (LDM) beamline at FERMI: Optical layout and first commissioning. J. Synchrotron Radiat. 2015, 22, 538-543.

37. Schäfers, F.; Mertins, H.C.; Gaupp, A.; Gudat, W.; Mertin, M.; Packe, I.; Schmolla, F.; Fonzo, S.D.; Soullié, G.; Jark, W.; et al. Soft-X-ray polarimeter with multilayer optics: Complete analysis of the polarization state of light. Appl. Opt. 1999, 38, 4074-4088.

38. Wang, H.; Dhesi, S.S.; Maccherozzi, F.; Cavill, S.; Shepherd, E.; Yuan, F.; Deshmukh, R.; Scott, S.; van der Laan, G.; Sawhney, K.J.S. High-precision soft X-ray polarimeter at Diamond Light Source. Rev. Sci. Instrum. 2011, 82, 123301.

39. Grizolli, W.; Laksman, J.; Hennies, F.; Jensen, B.N.; Nyholm, R.; Sankari, R. Multilayer based soft-X-ray polarimeter at MAX IV Laboratory. Rev. Sci. Instrum. 2016, 87, 025102.

40. Latimer, C.; MacDonald, M.; Finetti, P. A new method for polarization analysis in the VUV. J. Electron Spectrosc. Relat. Phenom. 1999, 101, 875-878.

41. Ilchen, M.; Glaser, L.; Scholz, F.; Walter, P.; Deinert, S.; Rothkirch, A.; Seltmann, J.; Viefhaus, J.; Decleva, P.; Langer, B.; et al. Angular Momentum Sensitive Two-Center Interference. Phys. Rev. Lett. 2014, 112, 023001.

42. Hartmann, G.; Lindahl, A.O.; Knie, A.; Hartmann, N.; Lutman, A.A.; MacArthur, J.P.; Shevchuk, I.; Buck, J.; Galler, A.; Glownia, J.M.; et al. Circular dichroism measurements at an X-ray free-electron laser with polarization control. Rev. Sci. Instrum. 2016, 87, 083113.

43. Codling, K.; Houlgate, R.G.; West, J.B.; Woodruff, P.R. Angular distribution and photoionization measurements on the 2p and 2s electrons in neon. J. Phys. B Atom. Mol. Phys. 1976, 9, L83.

44. Yao-Leclerc, I.; Brochet, S.; Chauvet, C.; De Oliveira, N.; Duval, J.P.; Gil, J.F.; Kubsky, S.; Lagarde, B.; Nahon, L.; Nicolas, F.; et al. Handling the carbon contamination issue at SOLEIL. Proc. SPIE 2011, 8077, 807712.

(C) 2017 by the authors. Licensee MDPI, Basel, Switzerland. This article is an open access article distributed under the terms and conditions of the Creative Commons Attribution (CC BY) license (http:/ / creativecommons.org/licenses/by/4.0/). 